\title{
Der politische »Fallout« von Nordkoreas Waffentests: Japans Sicherheitspolitik im Umbruch
}

\author{
Susanne Feske/Jan Helmig*
}

\begin{abstract}
The profound changes in Asia which have already been visible in shades clearly emerged in face of the North Korean test series. Especially for Japan the missile firings of July 2006 as well as the recent nuclear test have proved to be a trigger for a revised foreign policy posture. While it was formerly unthinkable to even discuss nuclear armament, such debate suddenly becomes a frequent part of political discourse, thus highlighting the growing military self-consciousness of Japan. To this end, an encompassing missile defense shield is a cornerstone of Japan's revised security attitude. This article traces how the regional concerns towards a Japanese return to a more assertive foreign policy stance were silenced by North Korea's nuclear test. It subsequently argues that despite the almost united protest against the North Korean provocation, the changing role of Japan's security posture meets with criticism, especially when procured under a missile defense shield. This constellation provides all the ingredients for an arms race in Asia.
\end{abstract}

Keywords: Raketenabwehr, Asien, Japan, Nordkorea, Rüstungswettlauf, Nuklearwaffen

\section{Einleitung}

Es ist also tatsächlich geschehen: Am 9. Oktober 2006, dem Tag der »nordkoreanischen Arbeiterpartei«, testete Nordkorea unterirdisch eine Atomwaffe mit einer geschätzten Sprengkraft von einer halben bis einer Kilotonne TNT. Seinen skurrilen Sinn für Symbolik hat Nordkorea einmal mehr bestätigt. Den Raketenstarts am 4. Juli 2006, dem amerikanischen Unabhängigkeitstag, folgte ein Atomtest am Tag der Wahl des zukünftigen südkoreanischen UN-Generalsekretärs Ban Ki Moon. Bereits ein Jahr zuvor hat Nordkorea seine Teilnahme an den so genannten Sechsergesprächen mit den USA, Japan, China, Südkorea und Russland über Pjöngjangs Atomprogramm aufgekündigt.

Im Vergleich zu den Bomben von Hiroshima und Nagasaki mit einer Sprengkraft von ca. 13 Kilotonnen nimmt sich der nordkoreanische Versuch eher bescheiden aus, und es ist ferner unklar, wie der Test technisch zu bewerten ist. ${ }^{1}$ Nach der internationalen Empörung als Reaktion auf die Tests und dem Beschluss der Vereinten Nationen, Sanktionen gegen Nordkorea zu verhängen, schlug Pjöngjang nicht etwa gemäßigtere Töne an, um die Situation zu deeskalieren. Ganz im Gegenteil: Die Regierung kündigte eine ganze Testserie an und erklärte ferner, dass amerikanische Sanktionen als Kriegserklärung interpretiert würden. ${ }^{2}$ Bereits jetzt zeichnen sich die weit reichenden Folgen ab, die die Nuklearpolitik Nordkoreas für die Sicherheitslage in Ostasien haben wird. Eine sicherheitspolitische Neuordnung war zwar bereits vor dem Test in Konturen erkennbar. Die zunehmende militärische Macht Chinas, verbunden mit einem neuen außenpolitischen Auftreten, die

\footnotetext{
* Susanne Feske ist Professorin an der Westfälischen Wilhelms-Universität Münster mit Schwerpunkt Asienpolitik. Jan Helmig promoviert an der Universität Bielefeld zur Geopolitik der amerikanischen Raketenabwehr. Dieser Aufsatz wurde referiert (peer-reviewed)

1 Während Nordkorea den Test als Erfolg verbucht, gehen amerikanische Experten davon aus, dass es nicht zu einer umfassenden Kettenreaktion gekommen ist. Partikel in der Atmosphäre weisen jedoch auf einen Atomtest hin. Richard L. Garwin/Frank N. von Hippel, A Technical Analysis of North Korea's Oct. 9 Nuclear Test, Arms Control Today, November 2006.

2 Nach UN-Sanktionen: Nordkorea droht mit "gnadenlosen Schlägen ", Süddeutsche.de (http://www.sueddeutsche.de/,tt2m1/ausland/artikel/ 906/88818/), 17. Oktober 2006.
}

wachsenden Instabilitäten auf der koreanischen Halbinsel, ungelöste territoriale Streitigkeiten ${ }^{3}$, der Aufbau eines regionalen Raketenabwehrsystems in Ostasien unter Federführung der USA und Japans ungeklärte Rolle als außenpolitisch »gefesselter Riese « ${ }^{4}$ lassen für die Zukunft zahlreiche einschneidende Veränderungen erkennen.

Der Atomwaffentest Nordkoreas hat den sicherheitspolitischen Verwerfungen in der Region eine neue Dimension hinzugefügt: die der atomaren Bedrohung und des atomaren Rüstungswettlaufs, an dem auch Japan beteiligt sein könnte. Zwar hat es schon immer Stellungnahmen einzelner Politiker und Militärs in Japan gegeben, dass das Land innerhalb kurzer Zeit eine Nuklearwaffe bauen könne und auch über die notwendigen Trägersysteme verfüge, um diese zu ihrem potenziellen Zielort zu transportieren. ${ }^{5}$ Jedoch stießen diese Äußerungen bisher auf ein überwiegend negatives Echo und führten, wie 1999, sogar zum Rücktritt des Vize-Verteidigungsministers. Bislang galt eine mögliche atomare Bewaffnung Japans - nicht nur aus historischer Perspektive - als politisch nahezu unmöglich. Die Folgen in der gesamten asiatisch-pazifischen Region wären unabsehbar und mit Sicherheit kontraproduktiv für Japan gewesen. Nun scheint allerdings dieses Tabu gebrochen. Jetzt ist in Kommentaren und Analysen zu hören, dass Japan nicht nur konventionell auf die Tests Nordkoreas antworten könnte, sondern auch mit einem eigenen Atomprogramm.

\footnotetext{
3 Gemeint ist damit der chinesische Anspruch auf das südchinesische Meer, insbesondere die Senkaku-Inseln zwischen Okinawa und Taiwan, der von Japan angefochten wird. Aufgrund vermuteter Ressourcenvorkommen als auch strategischer Zugangswege zu internationalen Märkten ist die Region für beide Parteien von Bedeutung. Vgl. Joachim Glaubitz, Japan und China: Zwischen Misstrauen und Kooperation, in: M. Pohl/H. J. Mayer (Hrsg.). Länderbericht Japan. Bundeszentrale für politische Bildung, Bonn 1998, S. 195-210, S. 205

4 Vgl. dazu beispielsweise P. Kevenhörster, Japans umfassende Sicherheit, Hamburg 2003 (zusammen mit D. Nabers).

5 Die zugeschriebene Zeitspanne variiert zwischen wenigen Wochen bis zur Jahresfrist. Zudem hat Japan, obwohl keine Atommacht, einen unabhängigen und geschlossenen nuklearen Brennstoffzyklus. Der einflussreiche Altpremier Nakasone forderte z.B. über Atomwaffen nachzudenken. Vgl. Florian Coulmas, Die virtuelle Atommacht, Die Zeit, Nr. 44, 26. Oktober 2006, S. 13. Auch der Chef der Internationalen Atomenergie-Organisation (IAEA), Mohamed El Baradei, spricht in diesem Zusammenhang von »virtuellen Atommächten «. Vgl. dazu (http://www.stern.de/politik/ausland/:IAEO30-Staaten-Atombombe/574117.html), 16.10.2006.
} 
Der neue japanische Ministerpräsident Abe beeilte sich zwar, solche Spekulationen entschieden zurückzuweisen. Sein Land würde niemals, so ließ er verlauten, Atomwaffen anschaffen oder fremde Atomwaffen auf seinem Territorium stationieren. ${ }^{6}$ Trotzdem wird deutlich: Über eine nukleare Option wird in Japan ernsthaft und offensiv diskutiert. Die Debatte über eine atomare Aufrüstung ist dabei nicht auf Japan begrenzt. Auch Südkorea hat nicht nur seine Truppen in Alarmbereitschaft versetzt, sondern auch öffentlich über die nukleare Option nachgedacht. China verfügt schon seit langem über Atomwaffen, jedoch kann auch hier eine qualitative und quantitative Aufwertung des Arsenals als Reaktion auf die nordkoreanische Testserie erwartet werden.

In diesem Artikel wird diskutiert, wie sich die Sicherheitslage in der asiatisch-pazifischen Region nach den Raketen- und Atomwaffentests Nordkoreas verändert hat. ${ }^{7}$ Im Mittelpunkt steht dabei Japan. Es wird argumentiert, dass bereits seit einigen Jahren Tendenzen in der japanischen Außen- und Sicherheitspolitik erkennbar sind, eine aktivere und offensivere militärische Rolle in der Region zu spielen. Entscheidend ist, dass die Opposition gegen eine solche Rolle Japans sowohl im Lande selbst als auch im regionalen Umfeld offensichtlich zum Schweigen gebracht worden ist. Denn angesichts der atomaren Bedrohung durch Nordkorea, gekoppelt mit dessen nicht zu unterschätzendem Raketenarsenal, werden Schritte akzeptabel, die vorher undenkbar erschienen. Für Japan gerät die nordkoreanische Testserie daher zum Katalysator für eine geänderte außen- und sicherheitspolitischen Haltung.

Zu den Bausteinen für ein offensives und militärisch aktiveres Japan gehören eine atomare Bewaffnung, der Aufbau eines regionalen Raketenabwehrsystems, die Neuinterpretation oder Streichung des Artikels 9 der Verfassung sowie eine gewandelte Auslegung des japanisch-amerikanischen Sicherheitsvertrages. Ersteres befindet sich, wie oben ausgeführt, zunächst noch im Diskussionsstadium und soll deshalb nicht im Mittelpunkt dieses Aufsatzes stehen. ${ }^{8}$ Letzteres ist bereits geschehen, z.B. in Gestalt der »Richtlinien der Verteidigungskooperation« vom September 1997, die eine aktivere Politik Japans im Falle regi-

6 Vgl. dazu auch die wiederholten Äußerungen des Vorsitzenden des Strategierats der Liberaldemokratischen Partei (LDP), Shoichi Nakagawa, über die Notwendigkeit einer umfassenden und lebhaften Debatte über eine nukleare Bewaffnung Japans, um dem nordkoreanischen Militärpotenzial eigene Abschreckungsmittel entgegen zu stellen (http://www.n-tv.de/721196.html), 15.10.2006. Zwar hat Abe in offiziellen Verlautbarungen in seiner Funktion als Regierungschef den Bestand des dreifachen »Nein« zu Atomwaffen bekräftigt. Äußerungen aus seiner Zeit als z.B. Regierungssprecher stehen dem jedoch entgegen. Vgl. dazu Florian Coulmas, Die virtuelle Atommacht, Die Zeit, Nr. 44, 26. Oktober 2006, S. 13.

7 Nach verschiedenen Berichten testete Nordkorea am 4. Juli 2006 wenigstens sieben Raketen. Davon eine Taepodong-2 Langstreckenrakete, über deren Reichweite zwar keine abschließenden Daten vorliegen, die nach Expertenmeinung jedoch sowohl Japan als auch Teile der USA erreichen kann. Während über Erfolg oder Misserfolg der Kurzstreckenraketen kaum etwas bekannt ist - außer, dass sie Hunderte Kilometer vor Japan ins Meer stürzten - gilt der Test der Langstreckenrakete als Fehlschlag. Weniger als eine Minute nach dem Start stürzte die Rakete ab und fiel zurück auf nordkoreanisches Territorium. Nordkorea bezeichnete die Versuche als erfolgreichen und wichtigen Baustein zur Verteidigungsfähigkeit des Landes. Weitere Tests wurden unterdessen angekündigt, um die »abschreckende Selbstverteidigung « auszubauen.

8 Auch wenn Premier Abe angekündigt hat, vorerst keine eigenen Atomprogramme zu initiieren, wird diese Option dennoch in außenpolitischen Zirkeln ernsthaft diskutiert. Es wird deutlich, dass auch der nordkoreanische Atomtest im Grunde unabhängig von seinem technologischen Erfolg weitreichende Konsequenzen hat. Ähnlich verhält es sich mit der umfassenden Diskussion japanischer Außenpolitik. onaler Krisen vorsehen. ${ }^{9}$ Die regionale Raketenabwehr ist von entscheidender Bedeutung für die Sicherheitsarchitektur der asiatisch-pazifischen Region. Regionale Raketenabwehr (TMD, Theatre Missile Defense) erscheint zunächst als ein defensives Konzept und somit geeignet, nukleare Erstschläge mit ballistischen Trägermitteln abzuwehren. Allerdings birgt ein Raketenschild immer auch eine implizite offensive Komponente, nämlich den Schutz des eigenen Territoriums bei gleichzeitig erhaltener Erstschlagskapazität. Durch eine effektive Raketenabwehr wird die Logik der Abschreckung ausgehebelt. Ein nuklearer Erstschlag provoziert einen Vergeltungsschlag der Gegenseite, gegen den nun ein Schutz besteht. Somit wird ein Erstschlag ohne die Zwangsläufigkeit des Gegenschlages auf das eigene Territorium möglich. Die Fähigkeit, aufsteigende Raketen abzufangen, verbunden mit einer eigenen atomaren Bewaffnung, verändert die strategische Gleichung in der Region und verschafft dem so ausgerüsteten Staat einen einseitigen strategischen Vorteil - ein Szenarium, das Japan zu einem unkalkulierbaren Faktor in der gesamten Region macht.

Zudem wird die Abschaffung oder zumindest Neuinterpretation des Artikels 9 der japanischen Verfassung diskutiert. Der Kriegsverzichtsartikel 9 besagt, dass Japan auf Krieg als souveränes Mittel zur Regelung internationaler Streitigkeiten für alle Zeiten verzichtet. Land-, See- und Luftstreitkräfte sowie andere Kriegsmittel werden nicht unterhalten. Zwar besitzt Japan Selbstverteidigungsstreitkräfte; diese sind aber gemäß des Verbotes der Wiederaufrüstung nicht (oder nur eingeschränkt) in der Lage, offensive oder strategische Operationen auszuführen. Nach strikter Lesart verbietet der pazifistische Artikel auch die Einbindung in kollektive Sicherheitsbündnisse und erlaubt nur individuelle Selbstverteidigung. Die militärische Zusammenarbeit mit den USA sowie US-Sicherheitsgarantien sind somit einseitig und binden Japan nicht an Beistandsverpflichtungen gegenüber den USA. De facto ist Artikel 9 jedoch bereits weitestgehend außer Kraft gesetzt worden. Aus Rücksicht auf die asiatischen Nachbarn werden die Buchstaben des Artikels jedoch nicht angetastet.

Die Revision des Artikels 9 in einem durch eine Raketenabwehr veränderten sicherheitspolitischem Gerüst birgt aber unzweifelhaft hohe Risiken. Eine Militarisierung und Prononcierung der japanischen Außenpolitik, noch dazu unter einem Raketenschild, wird die angespannte Lage in der Region weiter verschärfen. Nordkoreas Testserie birgt somit die große Gefahr, als Auslöser weitreichender Umstrukturierungen eine Rüstungsspirale in Gang zu setzen.

Um die Restrukturierung der japanischen Sicherheitspolitik angemessen nachvollziehen zu können, wird im Folgenden vor allem auf die unmittelbaren Reaktionen in der Region auf die nordkoreanische Testserie eingegangen. Da die Raketenabwehr in der japanischen Sicherheitspolitik eine entscheidende Rolle spielt, sollen die technischen Grundlagen dieses Systems anschließend skizziert werden. Nur auf dieser Basis ist dann eine politische Bewertung der japanischen Militärpolitik möglich.

\footnotetext{
9 Hans Jürgen Mayer, Japan und die USA: Eine Partnerschaft mit Haken und Ösen, in: M. Pohl/H. J. Mayer (Hrsg.). Länderbericht Japan. Bundeszentrale für politische Bildung, Bonn 1998, S. 184-194, S. 186.
} 


\section{Die japanischen Reaktionen - Verschärfung des Tons und sicherheitspolitische Konsequenzen}

Die Kritik an den nordkoreanischen Raketenstarts und dem Atomtest durch die internationale Staatengemeinschaft erfolgte umgehend, jedoch keineswegs unisono. Insbesondere die USA und Japan verurteilten die jeweiligen Tests scharf und verlangten nicht nur die Einstellung der Versuche, sondern auch die Einschaltung des UN-Sicherheitsrates. Als Antwort auf die Raketentests im Juli 2006 verhängte Japan zunächst moderate wirtschaftliche Sanktionen und untersagte z.B. nordkoreanischen Offiziellen die Einreise, setzte Charterflüge zwischen den Staaten aus und verwehrte Schiffen das Einlaufen in japanische Häfen. Zudem drohte Japan mit der Einstellung von Hilfsprogrammen und Nahrungsmittellieferungen. Im Zuge des Atomtests wurden diese Sanktionsmaßnahmen nochmals deutlich verschärft. Japan wartete dabei nicht die Stellungnahme des UN-Sicherheitsrates ab.

Noch deutlicher fiel die Antwort des japanischen Außenministers Taro Aso aus. Aso ließ verlauten, dass Japan das Recht habe, im Falle eines drohenden nordkoreanischen Angriffs präemptiv zu reagieren. Wenngleich Artikel 9 der japanischen Verfassung die Androhung oder die Anwendung von Gewalt untersagt, sieht der Außenminister angesichts der herrschenden Spannungen und unter dem Eindruck der Raketenstarts keinen Bruch mit der Verfassung. ${ }^{10}$ Damit provozierte die japanische Regierung prompte Reaktionen der umliegenden Nachbarn. Die aggressive Rhetorik Japans wurde mit großen Vorbehalten in Seoul und Beijing aufgenommen. ${ }^{11}$ So bezeichnete der südkoreanische Präsident Roh Moo-hyun die Androhung Japans, gegebenenfalls präemptiv einzugreifen, als »Überreaktion « und verwies darauf, dass die japanischen Äußerungen eine neue Situation geschaffen hätten, welche die gespannte Atmosphäre weiter anheizen könnte. ${ }^{12}$ Die Äußerungen des japanischen Verteidigungsminister Fukushiro Nukaga, der angesichts der nordkoreanischen Raketenstarts zudem über die Normalität begrenzter Angriffskapazitäten für souveräne Staaten sinnierte, sind darüber hinaus von allen Staaten in Asien mit Sorge registriert worden. ${ }^{13}$ Seoul hob daher hervor, dass die Spannungen nur mit bedachter Diplomatie gelöst werden könnten und widersetzte sich mit vorsichtigen Worten dem Druck der USA und Japans, die eine Resolution des UN-Sicherheitsrates forderten, um weitere Raketenstarts zu unterbinden.

Südkoreas mediative Politik die auf Ausgleich und Annäherung bedacht war ${ }^{14}$, hat sich spätestens mit dem nordkoreanischen Atomtest als unwirksam erwiesen. Auch von chinesischer Seite

10 Vgl. Agence France-Presse (AFP), Japan has Right to Protect Itself says Foreign Minister, (http://www.spacewar.com/reports/Japan_Has_Right_To_Protect_ Itself_Says_Foreign_Minister_999.html), 10.07.2006.

11 S. Tandon, North Korean Missile Drives Wedge through Asia, Agence France-Presse (AFP) (http://www.spacewar.com/reports/North_Korean_Missile_Drives_Wedge_Through_Asia_999.html), 10.07.2006.

12 South Korea Blasts Japanese Official's »Preemptive Strike« Remarks, VietNamNet, (http://english.vietnamnet.vn/international/2006/07/590363/), 12.07.2006.

13 Vgl. Agence France-Presse (AFP), Japan has Right to Protect Itself says Foreign Minister, (http://www.spacewar.com/reports/Japan_Has_Right_To_Protect_ Itself_Says_Foreign_Minister_999.html), 10.07.2006.

14 Vgl. dazu auch S. Feske, Amerikanische Hegemonialpolitik gegenüber Japan und Südkorea: Die Auseinandersetzungen um eine regionale Raketenabwehr, Raketenabwehrforschung International, Bulletin No. 36 (Winter 2002/03). wurde das Verhalten Pjöngjangs erst mit unterdrücktem, dann aber mit unverhohlenem Ärger gesehen. Zwar sind aus Beijing keine offenen Forderungen nach militärischen Sanktionen ergangen. Allerdings formulierte die chinesische Regierung Kritik an Nordkorea direkt an die Adresse Pjöngjangs und nicht, wie sonst üblich, über indirekte Kanäle. Das zum Zeitpunkt der Raketenabschüsse in der Region herrschende Unbehagen gegenüber einer offensiveren japanischen Haltung wurde durch den nordkoreanischen Atomtest zum Schweigen gebracht. Kritik an Äußerungen führender japanischer Politiker verhallte angesichts der nordkoreanischen Brüskierungen.

Als eine erste konkrete Konsequenz der nordkoreanischen Provokationen zeichnet sich bereits jetzt eine deutliche Beschleunigung bei der Umsetzung der japanischen Raketenabwehrprogramme ab. Die Raketenabwehr ist eines der zentralen Kooperationsprojekte zwischen Japan und den USA. Vorbehalte gegenüber dem Programm gründen auf Zweifeln an der technischen Durchführbarkeit von Raketenabwehrmaßnahmen, auf dem immensen finanziellen Aufwand, der dafür nötig ist, sowie auf der Sorge vor einer wachsenden Militarisierung und damit eines sicherheitspolitischen »blow backs «. ${ }^{15}$ Angesichts der nordkoreanischen Atomtests traten diese Vorbehalte jedoch schnell in den Hintergrund der Debatte. Zudem sind bereits Fakten geschaffen worden. Eine Abkehr von der technologischen Zusammenarbeit zwischen den USA und Japan im Bereich der Raketenabwehr erscheint zunehmend auch aus wirtschaftlichen Gründen wenig wahrscheinlich. Japanische Unternehmen werden Abfangraketen in Lizenz produzieren. Auch zeigt die japanische Industrie Interesse, an anderen High-Tech-Entwicklungen in der Raketenabwehr stärker beteiligt zu werden. Für die Bush-Regierung sind dies gute Nachrichten. ${ }^{16}$ Insbesondere angesichts der Mehrheitsverluste der Republikaner im Repräsentantenhaus bzw. im Kongress drohen die bis dato fließenden Finanzmittel zu versiegen. Lukrative Verträge zwischen den USA und Japan werden somit wahrscheinlich auch unter geänderten politischen Vorzeichen nicht angetastet; die Raketenabwehrprogramme würden auch im Falle eines nachfolgenden, eventuell skeptischeren demokratischen Präsidenten quasi institutionalisiert. ${ }^{17}$

Für die USA waren der Kampf gegen den Terror, der Einsatz im Irak sowie die Krisen im Nahen und Mittleren Osten lange Zeit wichtigste außenpolitische Themen. Nun rückt mit der nordkoreanischen Testserie das Thema der Raketenabwehr auf der politischen Agenda wieder nach vorne. Letztmalig sorgte das nordkoreanische Raketenprogramm 1998 für Schlagzeilen. ${ }^{18}$ Im so genannten Rumsfeld-Report wurde davor gewarnt, dass leichte, modifizierte Taepodong-Raketen Nordkoreas bis zu 10.000 Kilometer reichen und somit eine Gefährdung für das amerikanische Territorium darstellen könnten; für Japan bestünde diese Bedrohung ohnehin. Diese Langstreckenraketen

15 Mit blow back (zu dt.: Rückstoß) sind die unbeabsichtigten Konsequenzen gemeint, die als negative Folgen auf ein Verursacherland zurückfallen. Vgl. C. Johnson, Ein Imperium verfällt. Ist die Weltmacht USA am Ende? München 2001.

16 Sieff, Martin, Japan give Ballistic Missile Defense a Boost for Bush, United Press International, (http://www.spacewar.com/reports/Japan_Give_Ballistic_Missile_Defence_A_Boost_For_Bush_999.html), 06.10.2006.

17 Vgl. Ebd.

18 Vgl. H. Wulf, Poker um Nordkoreas Atomprogramm, Aus Politik und Zeitgeschichte (48/2005), S. $17 \mathrm{ff}$. 
könnte, so der Bericht, Nordkorea innerhalb von fünf Jahren bauen und testen, ohne dass die USA davon Kenntnis erlangen müssten. ${ }^{19}$ Ein kurze Zeit nach dem Erscheinen des Berichtes stattfindender Test schien alle Befürchtungen noch zu übertreffen. Eine mehrstufige nordkoreanische Langstreckenrakete überflog japanisches Territorium und stürzte anschließend, nach dem angeblichen Versagen einer Stufe, ins Meer. Da keine weiteren Tests folgten und nach den Anschlägen des 11. September 2001 das Problem des Terrorismus das wichtigste Thema auf der amerikanischen außenpolitischen Agenda wurde, trat die Diskussion um die Bedrohung durch Nordkorea in den Hintergrund. Gleichwohl ging die Entwicklung von Abwehrsystemen ungebremst weiter, und auch Nordkorea wurde von den USA in die Achse des Bösen eingereiht. Befürworter des TMD-Programms hatten es jedoch zunehmend schwer, die hohen finanziellen Aufwendungen für das Programm zu rechtfertigen. Hinzu kommen zahlreiche fehlgeschlagene Tests, die Zweifel an der technischen Durchführbarkeit des Programms berechtigt erscheinen lassen. Die nordkoreanischen Raketenversuche vom 4. Juli 2006 stärkten die Position der Raketenabwehr-Befürworter. Der Atomtest vom 9. Oktober 2006 hat die Befürchtungen außenpolitischer »Falken « bestätigt. ${ }^{20}$ Daher wird der Kritik an einer engen japanisch-amerikanischen Militärzusammenarbeit der Boden entzogen.

\section{Die Raketenabwehr}

Mit den ersten Aufklärungsberichten, die von aufgetankten nordkoreanischen Raketen berichteten, rückten auch Raketenabwehrprogramme wieder in das Zentrum des öffentlichen Interesses. Die Abwehr von Kurz- und Mittelstreckenraketen wird zumeist als »schauplatzgebundene « oder »taktische« Abwehr umschrieben. »Nationale« oder »strategische« Verteidigungsprogramme richten sich gegen Raketen mit Reichweiten von 5000 km oder mehr. Die hohen Geschwindigkeiten, aber auch die relativ geringe Größe der Objekte erschwert die Ortung anfliegender Raketen sowie die Unterscheidung zwischen einzelnen Teilen wie z.B. Gefechtskopf, Täuschkörpern oder Raketentrümmern. Dies gilt insbesondere für Langstreckenraketen, bei denen sich die Nutzlast von der eigentlichen Rakete im Verlauf des Fluges trennt. Dies resultiert in einer sehr kleinen sensorischen Signatur.

Durch die hohen Geschwindigkeiten der Objekte steht nur ein begrenzter Zeitraum für ein erfolgreiches Abfangen zur Verfügung. Automatisierte Abläufe und Kommunikation in Echtzeit zwischen den beteiligten Stellen sind daher unabdingbar. Zudem muss im Falle eines Angriffs mit einer Kurzstreckenrakete die Zerstörung des Ziels möglichst weit vom eigentlichen Zielgebiet entfernt erfolgen, um potenzielle Kollateralschäden

19 Vgl. Commission to Assess the Ballistic Missile Threat to the United States, Washington D.C., 15. Juli 1998. Die Studie ist weithin bekannt als »Rumsfeld Report «.

20 Womit selbstverständlich nicht gemeint ist, dass über Lösungsvorschläge nicht unvermindert kontrovers diskutiert wird. durch Trümmer oder den beschädigten Gefechtskopf zu vermeiden. ${ }^{21}$

Das Abfangen einer Rakete kann während drei zu unterscheidender Flugphasen geschehen. Während der ersten Phase erfolgen der Aufstieg und die Beschleunigung der Rakete auf der vorgegebenen Flugbahn. Ein Abfangen während der Antriebsphase bietet verschiedene Vorteile. Die Infrarot- und Radarsignatur des Flugkörpers ist z.B. gut zu lokalisieren, und die Rakete ist noch relativ langsam. Zudem sind eventuelle Gegenmaßnahmen wie Täuschkörper noch nicht eingesetzt. Bei einem erfolgreichen Treffer fallen die Trümmer der Rakete mit dem Gefechtskopf zurück auf feindliches Territorium. ${ }^{22}$ Nachteilig wirkt sich der begrenzte Zeitraum für die Entdeckung eines Starts und dem Beginn der Abwehrmaßnahmen aus. Es ist offensichtlich, dass die Abwehr während oder kurz nach dem Start nur wirksam sein kann, wenn Abwehrmaßnahmen in der Nähe feindlicher Abschussstellen positioniert sind.

Zwar steht beim Abfangen während der zweiten und mittleren Flugphase mehr Zeit zur Verfügung. Allerdings sind die Geschwindigkeiten wesentlich höher, und die Umweltbedingungen im Weltraum stellen hohe Anforderungen an die Technik. Ein Abfangen während der dritten und letzten Flugphase oder dem Wiedereintritt in die Atmosphäre birgt ebenfalls sowohl Vor- als auch Nachteile. Täuschkörper wie metallene Ballons bzw. flüssige oder feste Stoffe, die potenziell in der Lage sind, Sensoren in der mittleren Flugphase zu stören, werden vom tatsächlichen Gefechtskopf durch den Luftwiderstand beim Wiedereintritt in die Atmosphäre getrennt. Allerdings muss in diesem Fall das Abfangen des Gefechtskopfes schnell und zuverlässig erfolgen, da ein erneutes Angreifen aufgrund der geringen zur Verfügung stehenden Zeit kaum möglich ist. Durch potenzielle Kollateralschäden entstehen weitere Gefahren. ${ }^{23}$

Eine klare Trennung zwischen taktischer und strategischer Raketenabwehr ist akademisch. Insbesondere aufgrund der zugehörigen und notwendigen Kommunikationseinrichtungen lässt sich die Unterscheidung in einzelne Bereiche nicht ohne weiteres aufrechterhalten. Zudem lassen sich zahlreiche Komponenten, insbesondere bei der Bekämpfung unmittelbar nach dem Start, sowohl für taktische als auch strategische Raketen verwenden. Es erscheint daher treffender, nur noch von »einer« Raketenabwehr zu sprechen und die Differenzierung in trennbare Bereiche gänzlich aufzuheben. Konsequent hat die Bush-Administration daher die verschiedenen Programme unter der Ägide der Missile Defense Agency (MDA) konsolidiert. Die De-facto-Aufhebung der Dualität der Abwehrmaßnahmen ist keineswegs unbedeutend. Gerade strategische Verteidigungsfähigkeiten, noch dazu, wenn sie z.B. quasi »vor der

21 Theodor Postol, einer der prominentesten Kritiker von Raketenabwehrprogrammen argumentiert, dass während des Golfkrieges die meisten Schäden an israelischen Städten nicht durch die abgefeuerten irakischen Scud-Raketen verursacht wurden, sondern vielmehr durch fehlgeleitete Patriot-Abfangraketen. T. Postol, Lessons of the Gulf War Experience with Patriot, International Security, Vol. 16, No. 3 (Winter 1991/1992), S. 119-171.

22 Kritiker argumentieren allerdings, dass Trümmer und Gefechtskopf einer im Mittleren Osten abgefeuerten und sehr spät in der Antriebsphase zerstörten Rakete z.B. in der Türkei oder Mitteleuropa niedergehen könnten. Vgl. G. Forden, Laser Defenses: What if they Work? Bulletin of the Atomic Scientists, Vol. 58, No. 5 (2002), S. 48-53.

23 Eine Einführung in die grundsätzlichen technischen Konzepte und Systeme findet sich in J.M. Lindsay und M.E. O'Hanlon, Defending America. The Case for Limited National Missile Defense. Washington D.C. 2001, S. 29-115. 
Haustür « Chinas eingerichtet werden, berühren fundamentale Grundsätze der Abschreckungspolitik.

Neben zahlreichen nationalen Programmen arbeiten die USA mit verschiedenen Staaten an der Realisation des ehrgeizigen Verteidigungsprogramms. Abgesehen von der wahrscheinlichen Stationierung von Abwehrsystemen in Osteuropa, setzen die USA vor allem auf Japan große Hoffnung bei der Realisierung der anspruchsvollen Abwehrpläne. Für eine Profilschärfung japanischer Außenpolitik ist der sicherheitspolitische Rückhalt mittels einer Raketenabwehr essentiell.

\section{Raketenabwehr und die Veränderung der japanischen Sicherheitspolitik}

Wichtigster sicherheitspolitischer Partner der USA in Asien ist Japan. Es erscheint daher wenig überraschend, dass sich Japan an der Entwicklung eines Raketenabwehrschirmes beteiligt. Zudem wurde die japanische »Verteidigungslosigkeit « spätestens mit dem Testflug einer nordkoreanischen Rakete über japanisches Territorium 1998 deutlich. Der Erwerb von PAC-3 Patriot-Abfangraketen sowie die Zusammenarbeit mit eigenen, hochmodernen Aegis-Kreuzern in der Systemarchitektur der amerikanischen Raketenabwehr unterstreicht, welchen Stellenwert die Raketenabwehr für die japanische Sicherheitspolitik hat. Zudem unterzeichneten die USA und Japan Entwicklungsverträge, um auf Schiffen stationierte SM3-Langstreckenabfangraketen zu bauen. Das Joint-Venture der Entwicklung der SM-3-Raketen ist einer der wichtigsten Eckpunkte der japanisch-amerikanischen Raketenabwehr. Darüber hinaus haben die USA auf Honshu ein hochauflösendes $X$-Band-Radar zur Flugbahnerfassung ballistischer Raketen installiert. $^{24}$

Die Rüstungszusammenarbeit zwischen den USA und Japan wird sich unter dem Eindruck der nordkoreanischen Aufrüstung in Zukunft weiter vertiefen; die Wahl von Shinzo Abe zum Nachfolger Junichiro Koizumis als Ministerpräsident Japans wird diesen Trend noch verstärken. Der konservative Abe gehört dem rechten Flügel der japanischen Regierungspartei LDP an und gilt als außenpolitischer »Falke«. Er tritt dafür ein, die pazifistischen Grundsätze in der Verfassung abzuschwächen und eine emanzipiertere außenpolitische Haltung einzunehmen. ${ }^{25}$ Kernelement der Sicherheitspolitik ist für Abe eine starke Raketenabwehr. Auch weitere hochrangige Mitglieder der japanischen Regierung unterstreichen die zentrale Bedeutung, die der Raketenabwehr in der gesamten sicherheitspolitischen Konzeption beigemessen wird und fordern eine schnelle Umsetzung. »In addition to building monitoring radar networks, we would like to cooperate with the United States and put our joint missile interception into shape as quickly as possible, « so machte Verteidigungsminis-

24 Vgl. Juliana Gittler, U.S., Japan Opening a New Era of Missile Defense. Realignment Plan Includes Beefing up Ballistic Shield, Stars and Stripes, Pacific Edition, (http://www.stripes.com/article.asp?section=104\&article=34110\& archive=true), 12. Februar 2006.

25 Vgl. Koizumis Nachfolger. Shinzo Abe wird neuer Ministerpräsident in Japan, www.faz.net, 20. September 2006. ter Fukushiro Nukaga vor einem parlamentarischem Komitee die Ambitionen Japans deutlich. ${ }^{26}$

Außenminister Aso unterstrich zudem, dass die Bedeutung der nordkoreanischen Raketen nicht erst durch die Testserie 2006 als immens wahrgenommen wird. »Rodong missiles have been regarded as a direct threat to Japan since they were testfired in 1993. «Die daraus zu ziehenden Schlüsse folgten unmittelbar. »We are rushing to put an anti-ballistic missile system in place ahead of schedule. « ${ }^{27}$ Die nordkoreanischen Raketen- und Atomversuche beschleunigten die laufenden Kooperationsabkommen zwischen den USA und Japan nochmals deutlich. ${ }^{28}$ Nach vorangegangenen erfolgreichen Tests im Pazifik mit amerikanischen Abfangraketen, an denen auch ein japanisches Schiff beteiligt war, sowie der Unterzeichnung von langfristigen Kooperationsverträgen bei der Entwicklung gemeinsamer Abfangraketen, forderte der japanische Verteidigungsminister nicht nur die rasche Umsetzung des Programms, sondern erstmalig auch die Stationierung von modernen $P A C$ 3 Patriot-Abfangraketen auf amerikanischen Basen in Japan. ${ }^{29}$ Bis 2007 will Japan diese Kurzstreckenabfangraketen und bis 2008 SM-3-Raketen stationieren; letztere können auch gegen Langstreckenraketen eingesetzt werden. ${ }^{30}$

Der Zeitplan erscheint zumindest hinsichtlich der PAC-3-Raketen nicht unrealistisch. Ein amerikanisches Bataillon mit 24 Abwehrraketen wurde bereits nach Japan verlegt. Im März 2007 sollen die auf US-Stützpunkten stationierten PatriotRaketen schließlich einsatzbereit sein. Zusammen mit den seegestützten SM-3-Raketen, die Ziele auch außerhalb der Atmosphäre bekämpfen können, wäre dann eine zweigestaffelte Raketenabwehr implementiert.

Neben der Dislozierung eines Raketenschirmes werden zusätzliche sicherheitspolitische Konsequenzen diskutiert. So werden in Japan Rufe nach einem eigenen nuklearen Arsenal deutlich lauter. ${ }^{31}$ Dass Japan sowohl Spaltmaterial als auch die zugehörigen technischen Kapazitäten zum Bau einer Atombombe hat, ist unzweifelhaft. Bemerkenswert ist jedoch, dass

26 Japan's defense chief wants to speed up joint missile shield with US, MSN Mainichi Daily News, 06.07.2006.

27 Agence France-Presse (AFP), Japan has Right to Protect itself says Foreign Minister, (http://www.spacewar.com/reports/Japan_Has_Right_To_Protect_ Itself_Says_Foreign_Minister_999.html), 10.07.2006.

$28 \mathrm{Ob}$ die US-Raketenabwehr aber überhaupt in erster Instanz gegen Nordkorea gerichtet ist, wird von vielen Kritikern bezweifelt. Zudem wird häufig bemängelt, dass sich technische US-Einrichtungen wie die Positionierung von Radaranlagen nicht dazu eignen, nordkoreanische Raketenflugbahnen effektiv zu verfolgen. Die Bedrohung wird vielmehr, zumindest spiegelte dies die technische Disposition der Abwehrarchitektur wider, im Iran und in China gesehen. Nicht erst mit den gescheiterten Raketentests stellt sich nämlich die Frage, ob die nordkoreanische Rüstung die Leistungen zu vollbringen vermag, die ihr von außen zugeschrieben werden. Experten gehen zwar davon aus, dass Nordkorea waffenfähiges Plutonium besitzt, um sechs bis acht Sprengköpfe herzustellen. Ein Umstand, der zweifelsohne bedenklich genug ist. Aber damit ist keinesfalls sichergestellt, dass auch ein zugehöriger Sprengkopf konstruiert werden konnte; geschweige den eine miniaturisierte Version, die auf eine Langstreckenrakete passt. Dennoch müssen gerade die jüngsten Drohungen Nordkoreas ernst genommen werden, ist die Adaption von Sprengköpfen auf Raketen wohl eher eine Frage der Zeit denn eine Grundsatzentscheidung

29 Vgl. William Cole, Ship-Based Interceptor to be tested off Kaua'i, The Honolulu Advertiser, 21. Juni 2006; SpiegelOnline, USA stationieren Raketenabwehrsystem in Japan, (http://www.spiegel.de/politik/ausland/0,1518,427682,00. html), 20. Juli 2006.

30 Damit wird die angesprochene Zweischneidigkeit der Raketenabwehr sichtbar.

31 Vgl. Harumi Ozawa, North Korea Pushes Japan Further Down The Nuclear Path, Agence France-Presse, (http://www.spacewar.com/reports/North Korea_Pushes_Japan_Further_Down_Nuclear_Path_999.html), 05.10.2006 
die Debatte über eine ausgeweitete militärische Rolle Japans kaum noch auf nennenswerten Widerstand stößt. Eine atomare Abschreckung im pazifischen Raum muss allerdings nicht zwangsläufig auf japanischen Atomwaffen beruhen. Auch die Stationierung von amerikanischen Nuklearwaffen auf japanischem Territorium wird als Alternative diskutiert. Denkbar ist auch eine Arbeitsteilung zwischen den USA und Japan. Die so angestoßene Rüstungsspirale dreht sich maßgeblich um die Raketenabwehr. Jedoch wird es anders als zu Zeiten der Blockkonfrontation, kein »Gleichgewicht des Schreckens « geben. Die Konfliktlinien sind in Asien weitaus diffuser - es gibt nicht zwei Pole, die sich feindlich gegenüberstehen, sondern eine Vielzahl komplexer und miteinander verwobener Konfliktzentren. Sicher ist, dass eine effektive Raketenabwehr nur durch eine immer größere Anzahl von Raketen geschwächt werden kann, die den Raketenabwehrschirm gleichsam »löchrig «, also durchlässig machen. Sicher ist ebenso, dass die Veränderungen in Japans Sicherheitspolitik besonders von der Volkrepublik China und von Südkorea genauestens zur Kenntnis genommen und mit Sorge registriert werden.

Dass der neue japanische Ministerpräsident Abe Beijing und nicht Washington seinen ersten Auslandsbesuch abstattete, bedarf in diesem Zusammenhang der Interpretation. Ganz offensichtlich ist darin keine absichtliche Brüskierung der USA und damit verbunden eine Distanzierung von Washington zu sehen. ${ }^{32}$ Vielmehr ist davon auszugehen, dass dies mit Zustimmung Washingtons geschehen ist. Die amerikanische Regierung dürfte ebenso wie die neue Regierung unter Abe an einer Verbesserung der japanisch-chinesischen Beziehungen interessiert sein. Beide sind sich offensichtlich darin einig, dass China in eine Allianz gegen Nordkorea eingebunden werden muss, um dessen Isolierung voranzutreiben.

\section{Ausblick}

Am 31. Oktober 2006 kündigte die Regierung Nordkoreas an, die Sechsergespräche mit den USA, Japan, China, Südkorea und Russland wieder aufzunehmen. Damit sind die Spannungen auf der koreanischen Halbinsel und in der gesamten asiatisch-pazifischen Region zunächst entschärft. Dennoch hat Nordkorea mit seinen Atomtests Fakten geschaffen, deren Wirkung auch seine neue Verhandlungsbereitschaft keineswegs schwächt. Nordkorea kehrt als Atommacht an den Verhandlungstisch zurück und hat bereits klar gemacht, dass

32 Vorherige japanische Regierungschefs haben sich als erstes Ziel ihrer Antrittsreisen in der Regel Washington ausgesucht. es weitere Gespräche von der Rücknahme der Sanktionen abhängig machen wird. Die USA hingegen verbuchen das vermeintliche Einlenken Nordkoreas als außenpolitischen Sieg und verkündeten, die Rücknahme von Sanktionen vom Verhandlungsverlauf der Sechsergespräche abhängig zu machen. Was letztendlich den nordkoreanischen Positionswandel herbeigeführt hat, ist nicht vollständig klar. Plausibel erscheint, dass die Volksrepublik China, an deren wirtschaftlichem Tropf Nordkorea hängt, erheblichen Druck auf Pjöngjang ausgeübt hat, seine starre Haltung zu revidieren. Die einmal angestoßene Entwicklung der Rüstung und Gegenrüstung hat jedoch längst eine eigene Dynamik entwickelt, die nicht mehr aufzuhalten ist. Japan wird seine Sicherheits- und Militärpolitik in dem oben skizzierten Sinne auch nach der Wiederaufnahme der Gespräche weiterentwickeln. Die Bedrohung durch das nordkoreanische Waffen- und Atompotenzial bleibt unverändert bestehen. Zudem hat das sicherheitspolitische Umdenken in Japan lange vor den nordkoreanischen Atomtests begonnen. Schon seit mehreren Jahren versucht Japan ein eigenständiges außenpolitisches Profil zu entwickeln. Nach den Terroranschlägen in den USA vom September 2001 wurde Japans offensivere Außen- und Sicherheitspolitik international legitimiert und von den USA deutlich unterstützt. Spätestens damit ist ein Prozess in Gang gekommen, der durch die nordkoreanischen Tests allenfalls an Fahrt gewonnen hat. Darüber hinaus prägen auch die japanisch-chinesischen Animositäten die Ausrichtung der japanischen Sicherheitspolitik. Zwar sind beide Staaten durchaus bereit, in bestimmten und abgrenzbaren Sachgebieten miteinander zu kooperieren, wie die nordkoreanische Krise gezeigt hat. Dennoch sind die Spannungen zwischen Japan und China, die sich im Wesentlichen auf Japans Aggression gegenüber China vor und während des Zweiten Weltkrieges gründen, bei weitem nicht beigelegt, wie die jüngsten Auseinandersetzungen 2005 zeigen. Die chinesische Aufrüstung sowie seine rasante wirtschaftliche Entwicklung werden von Japan mit Besorgnis registriert. Nicht nur sieht Japan einen wirtschaftlichen Konkurrenten um die Vormachtsstellung in der Region, sondern auch durchaus eine potenzielle militärische Bedrohung, die im Übrigen auch in den USA als solche wahrgenommen wird. Öffentlich wird die militärische und politische Konkurrenz zwischen den beiden Ländern nicht thematisiert. Dennoch wird Japan mit Unterstützung der USA versuchen, ein Gegengewicht zur Dominanz der Volksrepublik China aufzubauen. Nordkoreas Verhandlungsbereitschaft hat also an der Rüstungsdynamik in der Region nichts geändert. Die nordkoreanischen Testserien erweisen sich somit als Katalysator für eine geänderte asiatische Sicherheitsarchitektur, deren Konturen sich immer deutlicher abzeichnen. 